
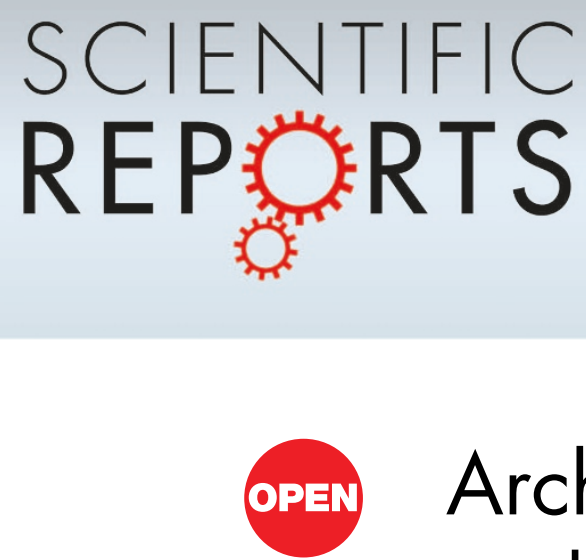

\title{
Architecture of the HCN selectivity filter and control of cation permeation
}

SUBJECT AREAS:

ION CHANNELS IN THE NERVOUS SYSTEM

NEUROPHYSIOLOGY

PERMEATION AND TRANSPORT

MEMBRANE BIOPHYSICS

Received

16 October 2012

Accepted

5 November 2012

Published

27 November 2012

Correspondence and requests for materials should be addressed to E.A.A. (eaaccili@mail. ubc.ca)

\author{
Vincenzo Macri, Damiano Angoli \& Eric A. Accili \\ Department of Cellular and Physiological Sciences, University of British Columbia, V6T 1Z3, Canada.
}

Hyperpolarization-activated Cyclic Nucleotide-modulated $(\mathrm{HCN})$ channels are similar in structure and function to voltage-gated potassium channels. Sequence similarity and functional analyses suggest that the HCN pore is potassium channel-like, consisting of a selectivity filter and an activation gate at the outer and inner ends, respectively. In GYG-containing potassium channels, the selectivity filter sequence is ' $T / S$ - $V / I /$ L/T-GYG', forming a row of four binding sites through which potassium ions flow. In HCNs, the equivalent residues are ' $C$-I-GYG', but whether they also form four cation binding sites is not known. Here, we focus on the anomalous filter residue of HCNs, the cysteine located at the inner side of the selectivity filter. In potassium channels, this position is occupied by threonine or serine and forms the fourth and most internal ion binding site of the selectivity filter. We find that this cysteine in HCNs does not contribute to permeation or form a fourth binding site. yperpolarization-activated Cyclic Nucleotide-modulated ( $\mathrm{HCNs}$ ) channels underlie the hyperpolarization-activated or funny current $\left(\mathrm{I}_{\mathrm{h}}, \mathrm{I}_{\mathrm{f}}\right)$, a critical regulator of membrane potential and spontaneous activity in cardiac myocytes and neurons $s^{1,2}$. HCNs possess a significant permeability to sodium, although potassium permeates to a much greater extent ${ }^{3}$. Sodium permeation is critical because it contributes to the depolarization of membrane potential following a strong hyperpolarizing stimulus. In addition to shifting the current reversal potential to less negative positive voltages, raising the concentration of extracellular potassium within a physiological or pathophysiological range of concentrations increases the conductance of $\mathrm{HCNs}^{3-6}$.

HCN channels are similar in structure and function to potassium-selective channels ${ }^{7,8}$. Like potassium channels, HCN channels pass predominantly potassium, are blocked by $\mathrm{mM}$ levels of cesium ions ${ }^{9-12}$ and their conductance is increased by extracellular potassium ${ }^{4,9,13-18}$. However, $\mathrm{HCN}$ channels are only minimally inhibited by barium, 4-aminopyridine or TEA ${ }^{3,13,19,20}$, which are strong blockers of potassium channels ${ }^{12}$. Because larger organic cations permeate ${ }^{20,21}$, the minimum diameter of the $\mathrm{HCN}$ pore may also be wider than that of potassium channels ${ }^{12,22}$. Finally, the single channel conductance of HCN channels is very small, less than 2 pS when measured in very high concentrations of potassium ${ }^{23,24}$; values of 5-50 pS have been determined for potassium channels measured at physiological potassium concentrations ${ }^{12}$. Structural determinants underlying differences between HCN and K-selective channels remain unknown.

A structure of a $\mathrm{HCN}$ channel pore has not yet been solved but the evidence supports a strong similarity to potassium channels. Like potassium channels, the primary sequence of HCNs predicts a pore consisting of the selectivity filter at the outer end and the voltage controlled gate at the inner side; the latter has been supported by functionally analyzing the accessibility of the pore to metals or drugs applied when the channels are open or closed $^{25-28}$. In GYG-containing potassium channels, the selectivity filter sequence is $T / S$-V/I/L/T-GYG, ${ }^{29,30}$ which form a row of four cation binding sites through which potassium ions move $\mathrm{e}^{22,31,32}$. In $\mathrm{HCNs}$, the equivalent residues are $C$-IGYG, but whether these also form four binding sites is not known.

Homology modeling and functional studies have proposed that the GYG residues and the innermost cysteine residues of the $\mathrm{HCN}$ selectivity filter face the intracellular medium ${ }^{25,28}$. Four cysteines are thought to form a ring around the internal opening of the selectivity filter, with their respective alpha carbons lying within $\sim 9 \AA$ and $\sim 6.5 \AA$, and their sulfur residues lying between $\sim 7 \AA$ and $\sim 10 \AA$, for opposite and adjacent residues, respectively. Functional support for this closeness among the inner cysteines comes from experiments showing that the application of cadmium from the cytoplasmic side irreversibly reduced the conductance of mouse HCN2 and sea urchin HCN channels in a way that implicates coordinated binding of this metal within the permeation path by four appropriately-spaced cysteine residues. Further support for such a close arrangement comes from reduction of $\mathrm{SpIH}$ conductance by intracellular application of the oxidizing agent copper phenanthroline, presumably because disulphide bonds are formed among the cysteines that block the permeation path. Reduction of conductance by either cadmium or copper phenanthroline did not occur in a mutant SpIH channel 
that, instead, contained an inner ring of serines; thus, in experiments using the wild type SpIH, the naturally occurring cysteines and the permeation path were likely targeted by those agents.

Even though the cysteine residues forming the inner ring may be close to each other, it remains an open question as to whether they impinge upon the permeation path itself to interact with permeating cations and form a fourth binding site. Knowledge of the number binding sites in $\mathrm{HCN}$ is important because this is has been shown to influence selectivity and gating of GYG-containing channels $\mathrm{s}^{33-35}$. Using the HCN2 isoform, we have used mutagenesis and electrophysiological methods to examine how near this inner ring of cysteines is to the permeation path. We find that the inner ring of cysteines has little impact on permeation or conduction, in support of a selectivity filter with only three cation binding sites.

\section{Results}

The cysteine 400 sulfhydryl side chain does not impact selectivity. To examine the role of cysteine 400 in selectivity, we characterized three substitutions of this residue in the mouse HCN2 channel. Serine and threonine were chosen, which are found naturally at this site in known potassium-selective channels (Fig. 1A). Each adds a hydroxyl group to a putative inner binding site of HCN2, although threonine has a larger volume $\left(116 \AA^{3}\right)$ as compared to serine $\left(89 \AA^{3}\right)$ by virtue of an additional $\mathrm{CH}_{3}$ group of its side chain. Alanine, with the approximate volume of serine, was also chosen as it effectively removes a charged side group yet does not likely alter the main-chain conformation or impose strong electrostatic or steric effects ${ }^{37}$. The volume of cysteine, the residue that is naturally present, is estimated to be $108.5 \AA^{3}$.

The HCN2 channel is permeable to both sodium and potassium ions ${ }^{7}$. For HCN2 channels expressed in Chinese hamster ovary $(\mathrm{CHO})$ cells, this can be appreciated from the point at which current reverses direction in instantaneous $\mathrm{I}_{\mathrm{f}}-\mathrm{V}$ plots, determined using solutions that contain physiological levels of sodium and potassium. To generate these plots, a pre-pulse to $-150 \mathrm{mV}$ was given to maximally activate the channels followed by test pulses to a series of less negative test voltages (Fig. 1B). The voltage protocol included a prior set of hyperpolarizing pulses to each test voltage from a holding potential of $-35 \mathrm{mV}$, to quantify the voltage-independent current existing at each test voltage prior to channel activation. The subtraction of voltage-independent current from instantaneous current measured after hyperpolarizing pre-pulse yields a measurement of instantaneous $\mathrm{I}_{\mathrm{f}}$, which is plotted against test voltage (Fig. 1C). Reversal potentials for the HCN2 channels used here were determined using solutions with physiological levels of sodium and potassium; this places the theoretical values of $\mathrm{E}_{\mathrm{Na}}$ and $\mathrm{E}_{\mathrm{K}}$ far apart to better reveal any differences between the mutant channels and the wild type channel.

As expected for $\mathrm{HCN} 2^{9,13}$, this plot crosses the $\mathrm{x}$-axis, or reverses, at $\sim-24 \mathrm{mV}$ under these conditions, in between the expected reversal potentials for $\mathrm{K}+$ and $\mathrm{Na}+$ calculated from the Nernst equation using physiological cation concentrations.

Transfection of HCN2 channels with alanine, serine and threonine substitutions produced robust HCN currents that were by and large the same as those produced with wild type HCN2. One notable difference was for the T400 channel, in which the rates of channel opening and closing were modified. Further experiments showed that the activation curve for the threonine substituted HCN2 channel was shifted to more positive potentials, by about $10 \mathrm{mV}$, and the rates of channel opening and closing were shifted and faster overall (Supplemental Fig. 1). Serine and alanine substitutions of C400 did not significantly impact reversal potential $\left(\mathrm{E}_{\mathrm{f}}\right)$, but the bulkier threonine significantly shifted $\mathrm{E}_{\mathrm{f}}$ to less negative values by $\sim 12 \mathrm{mV}$ (Fig. 1C, D).

Threonine at position 400 inhibits potassium conduction. The reduction in permeation and conduction of potassium relative to sodium by threonine at position 400 in the selectivity filter suggests that the bulkier side group of this residue, in contrast to the side groups of cysteine and serine, impinges upon the permeation path to modify cation flow. However, it is not clear if the threonine specifically inhibits potassium movement or increases sodium movement through the pore, or if both effects occur. To examine this, we measured and compared currents between wild type HCN2 and T400, using solutions that contained either potassium or sodium (at $135 \mathrm{mM}$, for both intracellular and extracellular solutions). To measure the current density upon full activation, we applied one 2 second hyperpolarizing pulse to $-150 \mathrm{mV}$ to $\mathrm{CHO}$ cells expressing either the wild type HCN2 or T400 channel (Fig. 2A). In potassium-only solutions, the current density was significantly larger for the wild type channel by $\sim 2$ fold compared to T400 (Fig. 2B).

We also examined conductance with intracellular and extracellular solutions containing only sodium because a threonine-induced increase in the permeation of this cation might have contributed to the greater $\mathrm{P}_{\mathrm{Na}} / \mathrm{P}_{\mathrm{K}}$ value. We were surprised to find that both the wild type HCN2 and T400 channels displayed robust hyperpolarizationactivated current (Fig 2A). Previous studies of cloned and native HCNs have uniformly suggested that current disappears in the absence of potassium ${ }^{7,38}$, suggesting that sodium is unable to permeate on its own. Current density in sodium-only solutions calculated for the HCN2 and T400 channels were not significantly different and considerably smaller than densities determined using potassium-only solutions (Fig. 2B). Together, the data suggest that the threonine side chain preferentially inhibits potassium movement. The reduction of potassium conduction, without a large change in sodium conduction, is consistent with the increase in calculated $\mathrm{P}_{\mathrm{Na}} /$ $\mathrm{P}_{\mathrm{K}}$ found above for the T400 mutant.

Elevation of conductance by extracellular sodium and potassium become close to equivalent in the T400 HCN2 channel. Previously, extracellular sodium was found to have no effect on the conductance of in vivo $\mathrm{HCN}^{2}$. More recently, using the $\mathrm{HCN} 2$ isoform expressed in HEK cells, extracellular sodium did enhance conduction but its effect was much smaller than that for extracellular potassium ${ }^{9}$; this suggests that enhancement may be linked to the relative abilities of sodium and potassium to permeate. However, the molecular mechanism underlying the effect of extracellular cations on conductance, and the difference between the effect of extracellular potassium and sodium, remain unknown. Because the relative permeation of sodium versus potassium was higher in the T400 HCN2 channel, we were able to examine this issue.

We first examined if the T400 channel conductance would increase to the same extent as the wild type $\mathrm{HCN} 2$ channel by raising the concentration of extracellular potassium in the absence of sodium. Raising extracellular potassium from a low $(5.4 \mathrm{mM})$ to a high concentration $(135 \mathrm{mM})$ increased current density by $\sim 9$ fold for both the wild type and T400 channels (Fig. 3). Thus, the degree of enhancement of conductance by extracellular potassium is maintained in the T400 mutant channel, despite an overall reduction in potassium conduction.

Because the conduction of sodium was already very small with a high concentration of extracellular sodium, we adopted a different approach to examine the effect of this cation on conductance. We examined the instantaneous I-V curve using reversed concentrations of potassium and sodium, without altering their combined total concentration. As we found previously for wild type $\mathrm{HCN} 2^{4,18}$, reversing the high concentration of sodium and low concentration of potassium for each not only shifted the reversal potential, but also increased the slope of this relation (Fig. 4A). Because the total concentration of permeating cations was unchanged, the data can be explained by an enhancement of conductance by extracellular potassium. For T400, the same protocol and solutions produced a much smaller change in slope conductance. The difference in current due 
to the change in solutions each test potential (Fig. 4B) clearly illustrates a much smaller increase in conductance produced by exchanging extracellular sodium and potassium concentrations. Thus, the effects of potassium and sodium on conductance are more similar in the mutant channel.
Block of the T400 channel by extracellular cesium supports a change in the permeation path. Together, the above evidence and the known primary structure of $\mathrm{HCNs}^{25,28}$ suggest that wild type HCN2 consists of three binding sites within the selectivity filter and that substitution of cysteine with threonine at position 400
A.

\begin{tabular}{|c|c|c|}
\hline HCN1 & $\mathrm{C}$ & IGYG \\
\hline HCN2 & C & $Y G$ \\
\hline HCN3 & $\mathrm{C}$ & y \\
\hline $\mathrm{HCN} 4$ & $\mathrm{C}$ & 10 \\
\hline Kir1.1 & $\mathrm{T}$ & IGY \\
\hline Kir2. 1 & $\mathrm{~T}$ & IC \\
\hline Kir3.1 & $\mathrm{T}$ & IGY \\
\hline KCNQ1 & $\mathrm{T}$ & IGY \\
\hline SK & S & IGY \\
\hline BK & $\mathrm{T}$ & VGY \\
\hline Shaker & $\mathrm{T}$ & VGY \\
\hline $\mathrm{Kv} 1.2$ & $\mathrm{~T}$ & \\
\hline Kv1. 5 & $\mathrm{~T}$ & VGY \\
\hline Kv2. 1 & $\mathrm{~T}$ & VG \\
\hline KVAP & $\mathrm{T}$ & VGY \\
\hline $\mathrm{Kcs} A$ & $\mathrm{~T}$ & V \\
\hline Mthk & $\mathrm{T}$ & VG \\
\hline Kv3.1 & $\mathrm{T}$ & LG \\
\hline $\mathrm{Kv} 4.2$ & $\mathrm{~T}$ & LG \\
\hline KAT1 & $\mathrm{T}$ & \\
\hline
\end{tabular}

C.

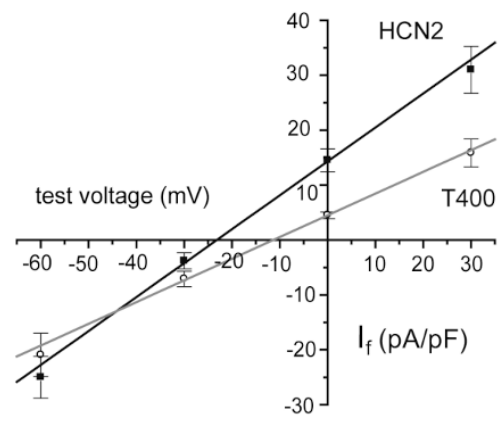

B.
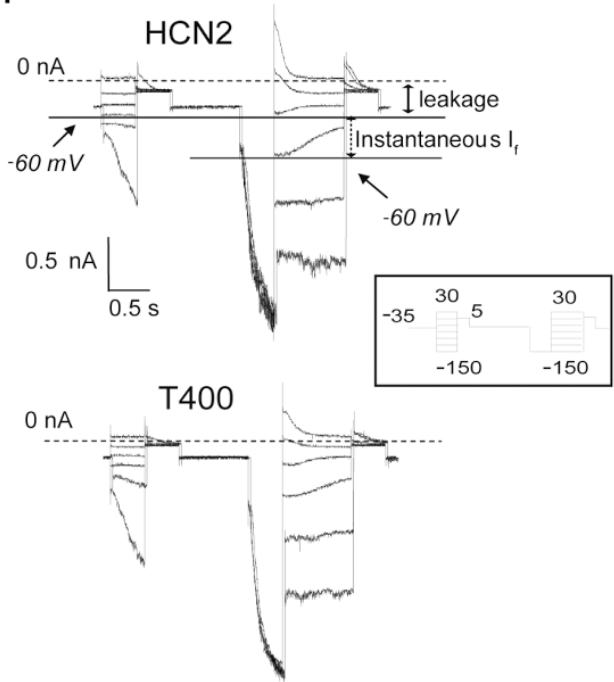

D.

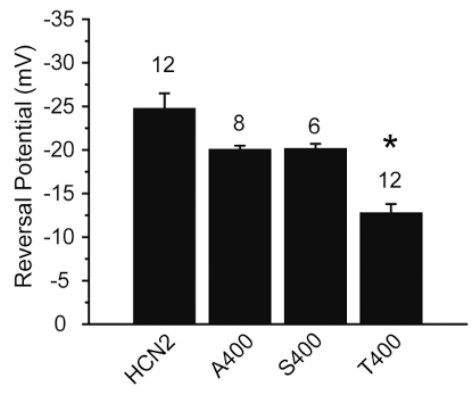

Figure $1 \mid$ Mutation of the innermost binding site from cysteine to threonine, but not to serine or alanine, alters reversal potential. (A) An alignment of the five amino acids forming the four cation binding sites of the selectivity filter of $\mathrm{K}+$ channels with those residues of the proposed selectivity filter of the four mammalian HCN channels. The conserved cysteines in HCN channels are highlighted in black. Note the conservation of the glycine-tyrosine-glycine 'GYG' motif and isoleucine among the $\mathrm{HCN}$ and $\mathrm{K}+$ channels, and the conservation of the threonine in all of the K+ channels except the SK channel. The amino acid sequences were aligned using ClustalW 1.8. (B) Current traces from two representative cells expressing HCN2 (upper) and T400 (lower) in response to an instantaneous $\mathrm{I}_{\mathrm{f}}-\mathrm{V}$ voltage protocol in a physiologic solution containing low potassium (5.4 mM) and high sodium (135 $\mathrm{mM}$ ) in the extracellular solution and high potassium $(130 \mathrm{mM})$ and low sodium $(10 \mathrm{mM})$ in the intracellular solution. $\mathrm{I}_{\mathrm{f}}$ is the slowly increasing component of current elicited in response to test voltage pulses, immediately following leakage current. The closed arrow highlights the leakage current at a test voltage of $-60 \mathrm{mV}$. The dashed line represents zero current. The open arrow highlights the instantaneous $\mathrm{I}_{\mathrm{f}}$ at a test voltage of $-60 \mathrm{mV}$, which follows a pre-pulse to $-150 \mathrm{mV}$. Instantaneous $\mathrm{I}_{\mathrm{f}}$ at each test voltage was calculated as the total instantaneous current at each test voltage, following a prepulse to $-150 \mathrm{mV}$, subtracted from the leakage current at that test voltage. The inset represents the voltage protocol used: a holding potential of $-35 \mathrm{mV}$, a series of initial test pulses from $-150 \mathrm{mV}$ to $30 \mathrm{mV}$, in $10 \mathrm{mV}$ increments, a subsequent pulse to $5 \mathrm{mV}$, back to the holding potential of $-35 \mathrm{mV}$, followed by another set of test pulses from $-150 \mathrm{mV}$ to $30 \mathrm{mV}$, another subsequent pulse to $5 \mathrm{mV}$, and back to the holding potential. The initial set of test pulses were used to estimate the leakage current at each test voltage. (C) Plots of instantaneous $\mathrm{I}_{\mathrm{f}}$ versus test voltage determined from the protocols in 'B'. Only the values for wild type HCN2 and T400 are shown in the plot. The solid lines represent linear fits through the mean values. (D) Bar graph comparing reversal potential between the wildtype $\mathrm{HCN} 2$ and the three $\mathrm{HCN} 2$ selectivity filter mutants. Reversal potentials were determined from instantaneous $\mathrm{I}_{\mathrm{f}}-\mathrm{V}$ plots of individual cells and averaged. The measured reversal potentials were $-24.6 \pm 1.8 \mathrm{mV}$ for HCN2 ( $\mathrm{n}=12$ cells, squares) and $-12.7 \pm 1.1 \mathrm{mV}$ for T400 $\left(\mathrm{n}=12\right.$ cells, circles), and were significantly different ( $\mathrm{t}$-test, $\mathrm{p}<0.05$, shown by asterisk in the figure). Permeability ratios $\left(\mathrm{P}_{\mathrm{Na}} / \mathrm{P}_{\mathrm{K}}\right)$ for the wild type and T400 channels were determined using the GHK equation (Equation 1) and were $0.35 \pm 0.02$ ( $\mathrm{n}=12$ cells) and $0.58 \pm 0.02$ ( $\mathrm{n}=12$ cells), respectively, and were also significantly different $(\mathrm{t}$-test; $\mathrm{p}<0.05)$. The same procedures were carried out using $\mathrm{S} 400$ and A400 mutant channels, which yielded reversal potentials of $-20.0 \pm 0.5 \mathrm{mV}(\mathrm{n}=8$ cells) and $-20.1 \pm 0.6 \mathrm{mV}(\mathrm{n}=6$ cells), respectively; these values were not significantly different from wild type ( $\mathrm{t}$-test, $\mathrm{p}>0.05)$. Values above the bars are the number of cells measured for each group. 
A.

$\mathrm{K}+$ only
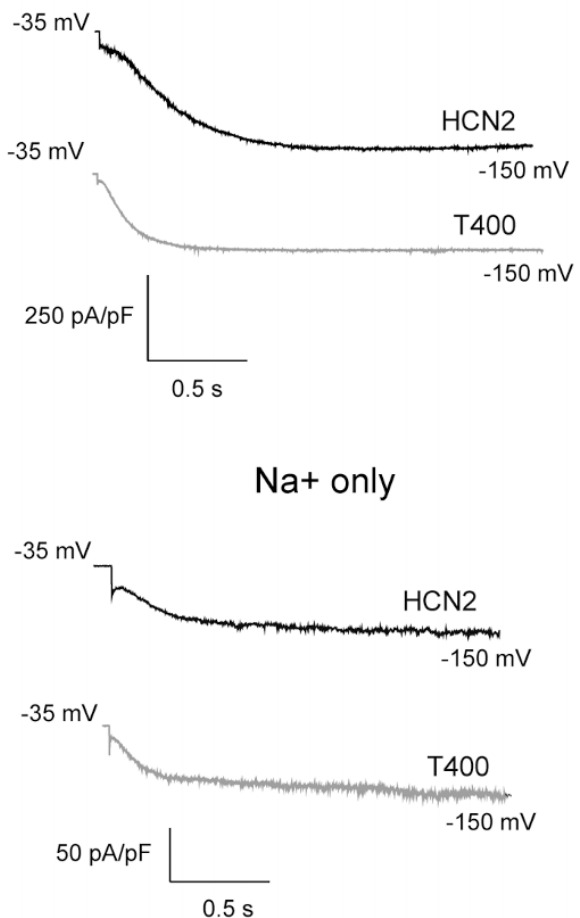

B.

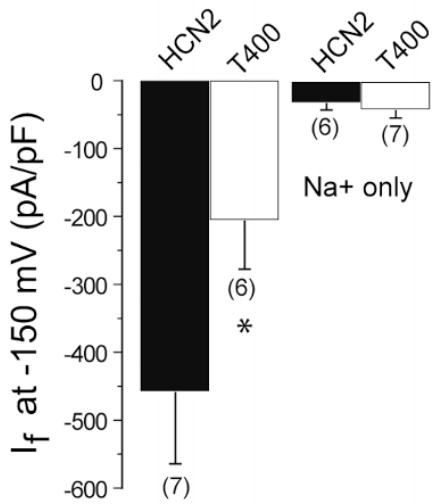

$\mathrm{K}+$ only

Figure $2 \mid$ The T400 mutation reduces potassium conduction. (A) HCN2 (black) and T400 (gray) current traces elicited at - $150 \mathrm{mV}$ for $2 \mathrm{~s}$, from a holding potential of $-35 \mathrm{mV}$ measured in symmetrical $(135 \mathrm{mM}$ ) potassium-only (top) or sodium-only (bottom) solutions. (B) Bar graph comparing current densities $(\mathrm{pA} / \mathrm{pF}$ ) of the HCN2 (black bar) and T400 (white bar) channels measured in potassium-only or sodium-only solutions. The numbers in parentheses represent the number of cells and the asterisk denotes a significant difference between HCN2 and T400 (t-test, $\mathrm{p}<0.05$ ).

alters the selectivity filter and permeation path. To further examine whether the permeation path is changed by threonine substitution, the inhibition of wild type and T400 channel conductance by extracellular cesium was examined. Cesium, which is larger than either sodium or potassium when dehydrated, is thought to bind within the ion conduction pathway of $\mathrm{HCNs}$ and obstruct cation flow, which becomes progressively greater at negative voltages ${ }^{6,9,18}$. Because we expected the bulkier side chains of threonine to either
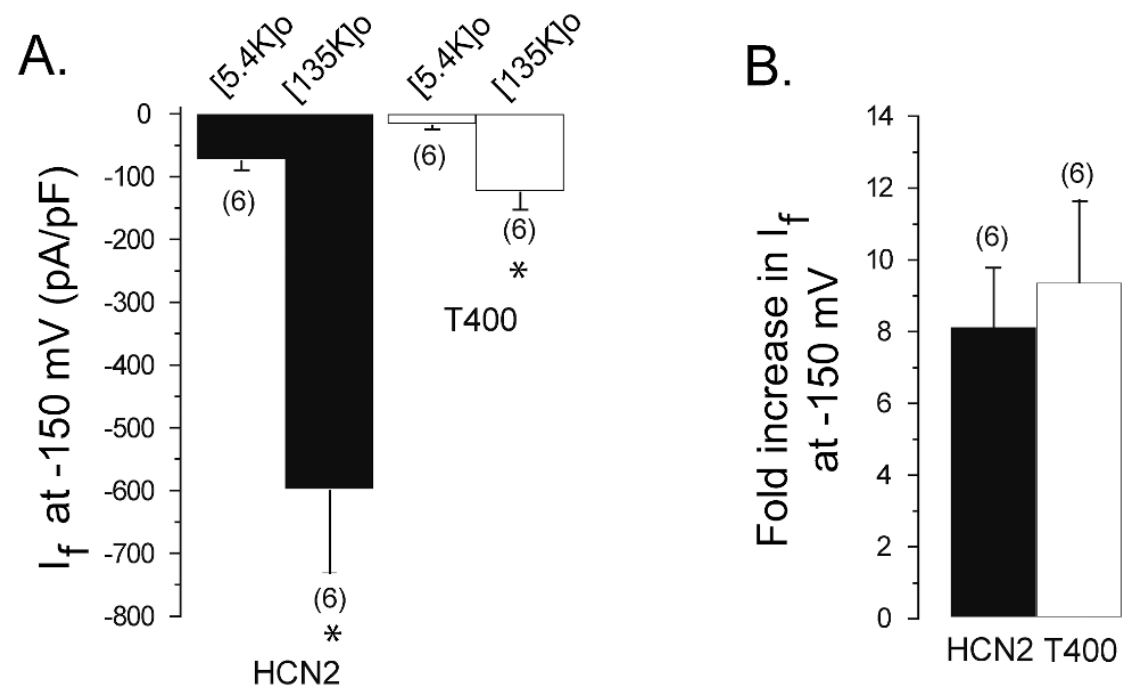

Figure 3 The T400 mutation has no effect on the degree of current enhancement by extracellular potassium. (A) Bar graph comparing wild type and T400 steady-state current density, in low $(5.4 \mathrm{mM})$ and high $(135 \mathrm{mM})$ concentrations of extracellular potassium, measured in the same cells in response to test pulses at $-150 \mathrm{mV}$, elicited from a holding potential of $-35 \mathrm{mV}$. The intracellular solution contained $130 \mathrm{mM} \mathrm{K}+$ and $10 \mathrm{mM} \mathrm{NMG}$. Asterisks denote significant difference between current density in low versus high extracellular potassium solutions ( $\mathrm{t}$-test, $\mathrm{p}<0.05)$. (B) Bar graph comparing the relative increase in current density of wild type and T400 when raising extracellular potassium from a low (5.4 mM K+/135 mM NMG) to high (135 mM $\mathrm{K}+/ 5.4 \mathrm{mM}$ NMG) concentrations, from " $\mathrm{A}$ ". There was no significant difference between in the fold-increase between wild type and mutant channels (t-test, $\mathrm{p}>0.05$ ). For both " $\mathrm{A}$ " and "B", the numbers in parentheses represent the number of cells measured. 
A.

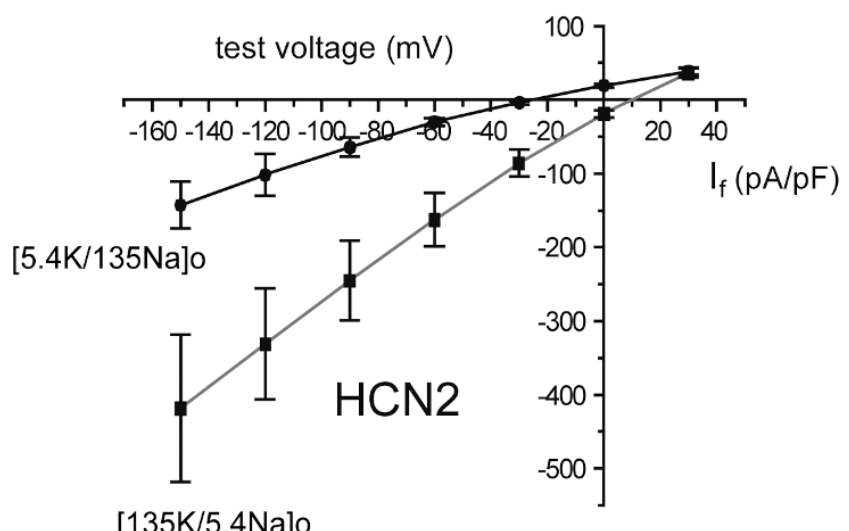

$[135 \mathrm{~K} / 5.4 \mathrm{Na}] \mathrm{o}$

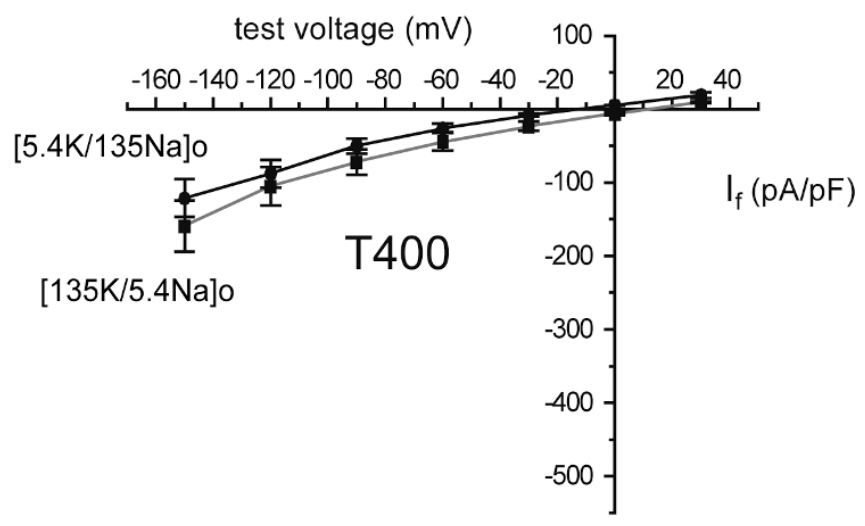

B.

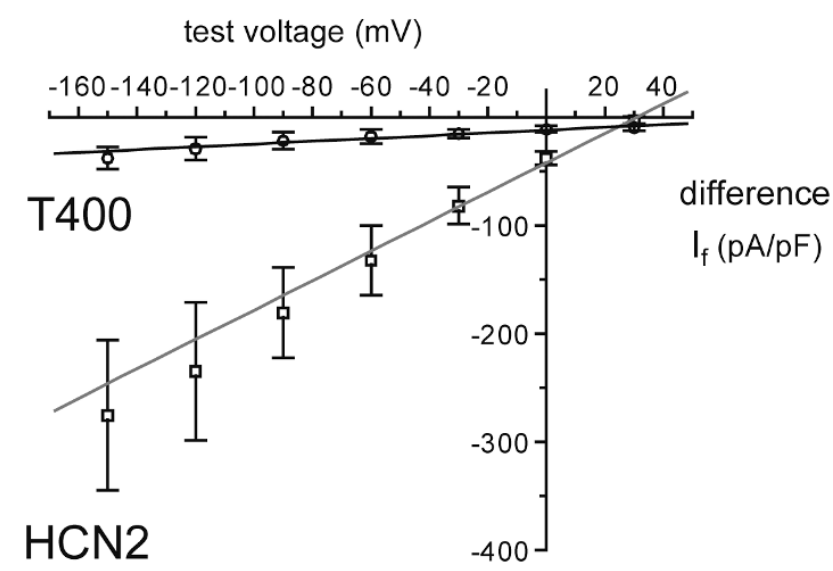

Figure $4 \mid$ The T400 mutation limits the increase in conductance produced by swapping extracellular sodium and potassium for each other. (A) Plots of instantaneous $\mathrm{I}_{\mathrm{f}}$ versus test voltages generated by protocols described in Figure $1 \mathrm{~B}$ at the indicated concentrations of potassium and sodium. The respective average $\mathrm{E}_{\mathrm{f}}$ and $\mathrm{P}_{\mathrm{Na}} / \mathrm{P}_{\mathrm{K}}$ values were $-25.5 \pm 2.6 \mathrm{mV}$ and $0.23 \pm 0.02$ (low extracellular $\mathrm{K}+$ ), and $10.0 \pm 1.0 \mathrm{mV}$ and $1.01 \pm 0.04$ (high extracellular $\mathrm{K}+)$ in the same cells expressing $\mathrm{HCN} 2\left(\mathrm{n}=6\right.$ cells). The average $\mathrm{E}_{\mathrm{f}}$ and $\mathrm{P}_{\mathrm{Na}} / \mathrm{P}_{\mathrm{K}}$ values were $-13.3 \pm 1.9 \mathrm{mV}$ and $0.39 \pm 0.02(\mathrm{low}$ extracellular $\mathrm{K}+$ ), and $9.6 \pm 2.2 \mathrm{mV}$ and $1.05 \pm 0.09$ (high extracellular $\mathrm{K}+$ ) in the cells expressing T400 ( $\mathrm{n}=6$ cells). In the low $\mathrm{K}+$ solution, the values for reversal potential were significantly different between cells expressing $\mathrm{HCN} 2$ and $\mathrm{T} 400(\mathrm{p}=0.0015)$ as were the $\mathrm{P}_{\mathrm{Na}} / \mathrm{P}_{\mathrm{K}}$ values $\left(\mathrm{p}=7.4 \times 10^{-4}\right)$. In the high $\mathrm{K}+$ solution, no significant difference in $\mathrm{E}_{\mathrm{f}}(\mathrm{p}=0.81)$ and $\mathrm{P}_{\mathrm{Na}} / \mathrm{P}_{\mathrm{K}}$ values $(\mathrm{p}=0.69)$ was seen between cells expressing HCN2 and T400. Within the same cell expressing either the mutant or wild type channel, the $\mathrm{E}_{\mathrm{f}}$ values were significantly different $\left(\mathrm{p}=9.9 \times 10^{-6}, \mathrm{p}=1.9 \times 10^{-7}\right.$, respectively) between low and high extracellular potassium. $\mathrm{P}_{\mathrm{Na}} / \mathrm{P}_{\mathrm{K}}$ values were also significantly different within cells expressing the mutant or wildtype channel $(\mathrm{p}=$ $6.5 \times 10^{-5}, \mathrm{p}=4.0 \times 10^{-8}$, respectively) between low and high extracellular potassium. Each cell was exposed to both low and high potassium solutions to remove variability in conductance that might have been due to differences in functional channel number and $\mathrm{E}_{\mathrm{f}}$ between cells. (B) Plots of the difference in instantaneous $\mathrm{I}_{\mathrm{f}}$ versus voltage produced in low and high $\mathrm{K}+$ solutions (determined from " $\mathrm{A}$ "). The line represents an average linear regression through the mean values of the difference in $\mathrm{I}_{\mathrm{f}}$ density recorded from the same cells in these two extracellular solutions. The slopes and correlation coefficients, determined by fitting of each cell individually, were averaged and the respective values were $1.55 \pm 0.39 \mathrm{nS}$ and $0.99 \pm 0.001$ for cells expressing HCN2, and $0.15 \pm 0.04 \mathrm{nS}$ and $0.86 \pm 0.04$ for cells expressing T400. Both the slopes $(\mathrm{p}=0.006)$ and the correlation coefficients $(\mathrm{p}=0.01)$ were significantly different between HCN2 and T400. 
form an additional site on the cytoplasmic aspect of the selectivity filter, or perturb the permeation path in some other way, we suspected that entry of Cs + and block conduction would be altered.

The effects of a wide range of Cs + concentrations on $\mathrm{I}_{\mathrm{f}}$ were determined from $\mathrm{CHO}$ cells expressing either HCN2 or T400 channels. An example of one concentration of Cs + utilized, $0.03 \mathrm{mM}$, on the instantaneous $\mathrm{I}_{\mathrm{f}}-\mathrm{V}$ curve is shown in Figure 5A. In the wild type channel, this concentration blocked a portion of the current only at the most negative potentials tested. In contrast, in the T400 channel, $0.03 \mathrm{mM}$ Cs + blocked a greater portion of the current and block appeared at less negative potentials.

To quantify the inhibition, the ratio of blocked and unblocked $I_{f}$ was calculated for each test voltage, plotted against Cs + concentration and fit with the Hill equation (Equation 2). In Fig. 5B, the plot for data collected at $-60 \mathrm{mV}$ shows that the mutant channel is blocked to a greater extent than the wild type channel over the same range of Cs + concentrations. To examine the voltage dependence of block of $\mathrm{I}_{\mathrm{f}}$ by cesium, values for $\mathrm{IC}_{50}$ were determined from the Hill equation, plotted against test voltage. The difference in these values between wild type $\mathrm{HCN} 2$ and T400 is striking; the former demonstrate notable dependence on voltage whereas the latter exhibit only a very subtle and scaled down dependence on voltage (Fig. $5 \mathrm{C}$ ). These data support perturbation of the permeation path by substituted threonine residue and suggest that block by Cs + in this mutant channel is strong but occurs with only minimal influence from the voltage field.

\section{Discussion}

To gain an understanding of the structure and function of the $\mathrm{HCN}$ selectivity filter, we examined a cysteine residue, found in place of serine or threonine that contributes to the innermost of four binding sites in potassium channels. Using the HCN2 isoform expressed in $\mathrm{CHO}$ cells, we show that replacement of this cysteine residue with alanine, or with serine, has no effect on reversal potential. Thus, the data suggest that the naturally-occurring sulfhydryl side chain of cysteine does not make contact with permeating ions or impact the environment near the cytoplasmic entrance to the filter. Threonine substitution also reduces potassium conductance, enhances blockade by $\mathrm{Cs}+$ and reduces the voltage-dependence of block by $\mathrm{Cs}+$. Together, the data suggest that threonine at position 400 either adds a fourth binding site to the HCN2 selectivity filter or it disrupts three existing binding sites; in either case, and together with other data, they support close proximity between the naturally-occurring cysteine at this position and the permeation path, without a significant interaction of the sulfhydryl groups on the permeation process itself.

Our results offer insight into the increase in $\mathrm{HCN}$ whole-cell conductance induced by raising extracellular potassium. $\mathrm{HCN}$
A.

\section{HCN2}

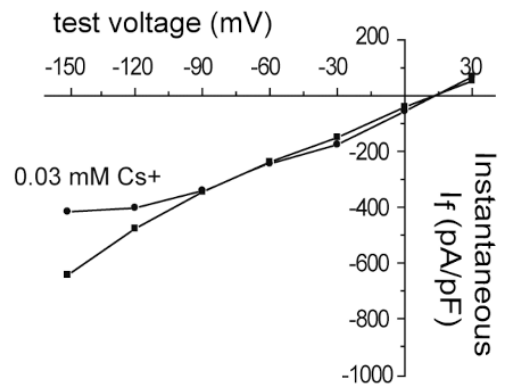

B.

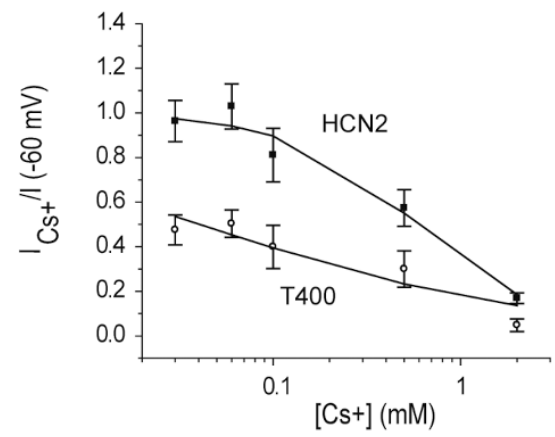

T400

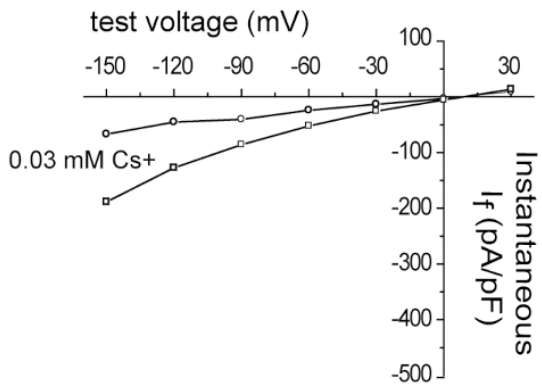

C.

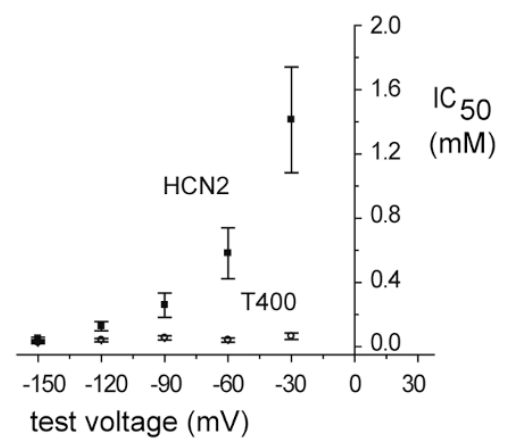

Figure $5 \mid$ Compared to its effect on the wild type HCN2 channel extracellular Cs + blocks the T400 channel with greater sensitivity and lesser dependence on voltage. (A) Plots of instantaneous $\mathrm{I}_{\mathrm{f}}$ versus voltage in cells expressing $\mathrm{HCN} 2$ or T400, determined using the voltage protocol and analysis described in Fig. 1, before and during perfusion with $0.03 \mathrm{mM} \mathrm{Cs}^{+}$with $135 \mathrm{mM} \mathrm{K}+$ and $5.4 \mathrm{mM} \mathrm{Na}+$ in the extracellular solution and $130 \mathrm{mM} \mathrm{K}+$ and $10 \mathrm{mM} \mathrm{Na}+$ in the intracellular solution $\left(\mathrm{HCN} 2\right.$, filled squares, $\mathrm{HCN} 2+\mathrm{Cs}^{+}$, filled circles, $\mathrm{n}=5$ cells; $\mathrm{T} 400$ open squares, $\mathrm{T} 400+\mathrm{Cs}^{+}$, open circles, $\mathrm{n}=$ 6 cells). This type of experiment was carried out for a range of Cs + concentrations and test voltages, from which the ratio of blocked current was determined and plotted against Cs + concentration (example for $-60 \mathrm{mV}$ shown in "B", below). (B) Plot of the ratio of blocked current at $-60 \mathrm{mV}$, obtained from instantaneous $\mathrm{I}_{\mathrm{f}}-\mathrm{V}$ curves as shown in " $\mathrm{A}$ ", versus Cs + concentration, for HCN2 (filled squares) and T400 (open circles). The values for the ratio of blocked current represent means \pm s.e.m. Solid lines represent fits of the data with the Hill equation (Equation 2), which gave values for $\mathrm{IC}_{50}$ and Hill factor (n); this analysis was carried out for a range of test voltages from which IC50 values were obtained and plotted against voltage ("C"), below). (C) Plot of $\mathrm{IC}_{50}$ values, obtained from Hill plots as determined in "B", versus test voltage for HCN2 (filled squares) and T400 (open circles) channels. The $\mathrm{IC}_{50}$ values represent means \pm s.e.m. 
conductance is steeply sensitive within a range of potassium concentrations found in vivo ${ }^{4-6,39-41}$, and so this effect likely contributes to naturally occurring changes in cellular electrical activity. Most previous studies of in vivo $\mathrm{HCN}$ have shown that extracellular sodium has little effect on conductance ${ }^{2}$. However, one study using the HCN2 isoform expressed in HEK cells showed that extracellular sodium did enhance conduction but its effect was much smaller than that for extracellular potassium 9 . Here, raising extracellular potassium from a low to a high level in the absence of sodium significantly enhanced conductance at $-150 \mathrm{mV}$ by $\sim 9 \times$ in both the wild type and T400 mutant channel; this implies that the degree of enhancement of conductance produced by extracellular potassium is not regulated by the inner selectivity filter and is independent of ion turnover in the pore per se. Furthermore, conductance of T400 was only minimally altered when high levels of extracellular sodium were replaced with potassium, implying that conductance remained almost equally elevated in both conditions. Together, the data suggest that the smaller enhancement of conductance by sodium compared to potassium found previously in wild type $\mathrm{HCN} 2^{9}$ is mainly due to a downstream difference in ion turnover in the pore rather than to a difference in degree of enhancement between these two cations.

We were surprised to find robust expression of $\mathrm{I}_{\mathrm{f}}$ in $\mathrm{CHO}$ cells containing wild type $\mathrm{HCN} 2$ channels with only sodium in the intracellular and extracellular solutions. In contrast to this finding in our study, many previous studies have suggested that potassium on the same side of the membrane is required in order for sodium to permeate HCN channels e.g. ${ }^{2,3,9,13}$. For both wild type and T400 channels, the current density in sodium-only solutions was $\sim 25 \mathrm{pA} / \mathrm{pF}$ (at $-150 \mathrm{mV}$ ), much smaller than the potassium-only currents that were $\sim 450 \mathrm{pA} / \mathrm{pF}$. Because of low density at a very negative membrane potential, sodium-only currents may have gone undetected in previous studies.

Substitution of cysteine 400 in HCN2 with threonine recapitulates a potassium channel selectivity filter, but it did not confer high selectivity for potassium. This is not surprising since, in potassium channels, mutation of this threonine to several other amino acids does not render them less selective for potassium ${ }^{12,42}$. Moreover, inwardly rectifying channels with an intact selectivity filter, but with a pore helix mutation that faces the internal cavity, lack potassium selectivity; further addition of charged residues in the cavity restore potassium selectivity ${ }^{43-45}$. Finally, studies of non-selective NaK channel have shown that the TVGYG sequence forms four inline binding sites that are pre-requisite for high potassium selectivity but that other factors and interactions are also necessary ${ }^{33,35,46}$.

A hint of what the HCN pore might look like is offered by a crystallographic study of the KcsA potassium channel from Streptomyces lividans showing that substitution of the threonine with cysteine at the inner site of the selectivity filter (the reverse of what we did here) results in the removal of the hydroxyl group and replacement of the $\gamma$-carbon of the threonine side-chain with the sulfur side group such that it faces away from the permeating path; this opens up the vestibule and dramatically reduces potassium binding at this site ${ }^{34}$. The KcsA structure was otherwise unaltered by the cysteine substitution, with the backbone carbonyl groups that form the first three sites of the selectivity filter remaining at 3-4 $\AA$ apart. It remains to be seen whether direct observation of the $\mathrm{HCN}$ selectivity filter, for example by X-ray crystallography, will support a similar three site selectivity filter and the cysteine residues lying close together, but with the sulfur side chains facing away from the permeation path.

\section{Methods}

Site-directed mutagenesis. Three selectivity filter mutant channels, HCN2 C400T IGYG (T400), HCN2 C400S-IGYG (S400) and HCN2 C400A-IGYG (A400), were constructed by overlapping PCR mutagenesis from a mouse HCN2 template as previously described ${ }^{4}$. The amplified mutagenized products were subsequently digested with NheI and BlpI and ligated within the complementary wild type HCN2 vector. The mutations were confirmed by restriction enzyme analysis and automated sequencing (carried out at The Centre for Molecular Medicine and Therapeutics, DNA Sequencing Core Facility, BC Children's and Women's Hospital, University of British Columbia, Vancouver Canada).

Tissue culture and expression of $\mathrm{HCN} 2$ constructs. Chinese Hamster Ovary $(\mathrm{CHO})$ cells (ATCC, Manassas, VA) were maintained in Hams F-12 media supplemented with antibiotics and 10\% FBS (Gibco, Burlington, Ontario), and incubated at $37^{\circ} \mathrm{C}$ with $5 \% \mathrm{CO}_{2}$. Cells were plated onto glass coverslips. Two days after splitting, mammalian expression vectors encoding wild type or mutant HCN2 channels ( $2 \mu \mathrm{g}$ per $35 \mathrm{~mm}$ dish), and a green fluorescent protein (GFP) reporter plasmid ( $0.6 \mu \mathrm{g}$ per dish) were transiently co-transfected into the cells using the FuGene6 transfection reagent (Roche Biochemical, Indianapolis, IN).

Whole-cell patch clamp electrophysiology. CHO Cells expressing GFP were chosen for whole-cell patch clamp recordings $24-48$ hours post transfection. The pipette solution contained varying concentrations of $\mathrm{K}$ aspartate, $\mathrm{NaCl}$ or $\mathrm{N}$-methyl Dglucamine (NMG) (see figure legends for each experimental condition) with each solution containing, $0.5 \mathrm{mM} \mathrm{MgCl}_{2}$, 1 mM EGTA, 5 mM HEPES, $\mathrm{pH}$ adjusted to 7.4 with $\mathrm{KOH}$ or $\mathrm{NaOH}$ depending upon the experimental condition. The extracellular solution contained varying concentrations of $\mathrm{NaCl}, \mathrm{KCl}$, and $\mathrm{NMG}$ (see figure legends for each experimental condition) with each solution containing, $1.8 \mathrm{mM}$ $\mathrm{CaCl}_{2}, 0.5 \mathrm{mM} \mathrm{MgCl}_{2}, 5 \mathrm{mM}$ HEPES, pH adjusted to 7.4 with $\mathrm{KOH}$ or $\mathrm{NaOH}$ depending upon experimental condition. Whole-cell currents were recorded using an Axopatch 200B amplifier and Clampex software (Axon Instruments, Union City, CA) at room temperature $\left(20-22^{\circ} \mathrm{C}\right)$. Patch clamp pipettes were pulled from borosilicate glass and fire polished before use (pipette $\mathrm{R}=2.5-4.5 \mathrm{M} \Omega$ ).

Data analysis. Data were filtered at $2 \mathrm{kHz}$ and were analyzed using Clampfit (Axon Instruments, Union City, CA), Origin (Microcal, Northhampton, MA) and Excel (Microsoft, Seattle, WA) software. Instantaneous $\mathrm{I}_{\mathrm{f}}-\mathrm{V}$ relations were generated as described in our previous studies of $\mathrm{HCN}$ channels e.g. ${ }^{18,36}$ which were used determine the reversal potential for $\mathrm{HCN}$-mediated $\mathrm{I}_{\mathrm{f}}\left(\mathrm{E}_{\mathrm{f}}\right)$. Briefly, a two part protocol was utilized. First, $500 \mathrm{~ms}$ test pulses ranging from $+30 \mathrm{mV}$ to $-150 \mathrm{mV}$, from a holding potential of $-35 \mathrm{mV}$, were used to determine the amplitude of leakage currents at each test voltage. Second, a $500 \mathrm{~ms}$ pre-pulse to $-150 \mathrm{mV}$ from a holding potential of $-35 \mathrm{mV}$, to open the channels, was followed by test potentials ranging from

$+30 \mathrm{mV}$ to $-150 \mathrm{mV}$ to determine total instantaneous currents at each test voltage. The pre-pulse length was kept to $500 \mathrm{~ms}$ to minimize ionic fluxes that could occur over the course of the experiment in a single cell. The leakage current, was subtracted from the total instantaneous current at each test voltage to yield values of instantaneous $I_{f}$, which were plotted against test voltage to determine $E_{f}$ which is the point that crosses the $\mathrm{x}$-axis. $\mathrm{E}_{\mathrm{f}}$ values were used to determine permeability ratios for $\mathrm{Na}^{+}$and $\mathrm{K}^{+}\left(\mathrm{P}_{\mathrm{Na}} / \mathrm{P}_{\mathrm{K}}\right)$ using the following Goldman Hodgkin Katz equation as described previously for $\mathrm{HCN}$ channels ${ }^{9}$,

$$
\mathrm{E}_{\mathrm{f}}=(\mathrm{RT} / \mathrm{F}) \ln \left(\left[\mathrm{K}_{\mathrm{o}}+\left(\mathrm{P}_{\mathrm{Na}} / \mathrm{P}_{\mathrm{K}}\right) \mathrm{Na}_{\mathrm{o}}\right] /\left[\mathrm{K}_{\mathrm{i}}+\left(\mathrm{P}_{\mathrm{Na}} / \mathrm{P}_{\mathrm{K}}\right) \mathrm{Na}_{\mathrm{i}}\right]\right)
$$

In order to examine block of $\mathrm{I}_{\mathrm{f}}$ block by extracellular $\mathrm{Cs}^{+}$in $\mathrm{CHO}$ cells expressing HCN2 or T400, we used the Hill equation were used. Cs + dose-response curves (Fig. 3C) were measured using various concentrations of extracellular Cs + and voltages and fitted with the Hill equation,

$$
\mathrm{I}_{\mathrm{Cs}+} / \mathrm{I}=1 / 1+\left([\mathrm{Cs}+] / \mathrm{IC}_{50}\right)^{\mathrm{n}}
$$

where the $\mathrm{IC}_{50}$ is the concentration at which half of the channels are blocked and " $n$ " is the cooperativity factor between Cs + and the blocking site. Both the wild type and mutant channels had $\mathrm{n}$ values near 1 . The $\mathrm{IC}_{50}$ values were then plotted against test voltage.

All the experiments and procedures were approved by the Office of Research Services, University of British Columbia.

1. DiFrancesco, D. Pacemaker mechanisms in cardiac tissue. Annu Rev Physiol 55, 455-72 (1993).

2. Pape, H. C. Queer current and pacemaker: the hyperpolarization-activated cation current in neurons. Annu Rev Physiol 58, 299-327 (1996).

3. DiFrancesco, D. A study of the ionic nature of the pace-maker current in calf Purkinje fibres. J Physiol 314, 377-93 (1981).

4. Macri, V., Proenza, C., Agranovich, E., Angoli, D. \& Accili, E. A. Separable gating mechanisms in a Mammalian pacemaker channel. J Biol Chem 277, 35939-46 (2002).

5. Solomon, J. S. \& Nerbonne, J. M. Hyperpolarization-activated currents in isolated superior colliculus-projecting neurons from rat visual cortex. J Physiol 462 393-420 (1993)

6. DiFrancesco, D. Block and activation of the pace-maker channel in calf purkinje fibres: effects of potassium, caesium and rubidium. J Physiol 329, 485-507 (1982).

7. Biel, M., Wahl-Schott, C., Michalakis, S. \& Zong, X. Hyperpolarization-activated cation channels: from genes to function. Physiol Rev 89, 847-85 (2009).

8. Robinson, R. B. \& Siegelbaum, S. A. Hyperpolarization-activated cation currents: from molecules to physiological function. Annu Rev Physiol 65, 453-80 (2003). 
9. Moroni, A. et al. Kinetic and ionic properties of the human HCN2 pacemaker channel. Pflugers Arch 439, 618-26 (2000).

10. Ludwig, A. et al. Two pacemaker channels from human heart with profoundly different activation kinetics. Embo J 18, 2323-9 (1999).

11. Mistrik, P. et al. The murine HCN3 gene encodes a hyperpolarization-activated cation channel with slow kinetics and unique response to cyclic nucleotides. J Biol Chem 280, 27056-61 (2005).

12. Hille, B. Ionic channels of excitable membranes.

13. Ludwig, A., Zong, X., Jeglitsch, M., Hofmann, F. \& Biel, M. A family of hyperpolarization-activated mammalian cation channels. Nature 393, 587-91 (1998).

14. Yang, Y. \& Sigworth, F. J. Single-channel properties of IKs potassium channels. J Gen Physiol 112, 665-78 (1998).

15. Sakmann, B. \& Trube, G. Conductance properties of single inwardly rectifying potassium channels in ventricular cells from guinea-pig heart. J Physiol 347, 641-57 (1984).

16. Stampe, P., Arreola, J., Perez-Cornejo, P. \& Begenisich, T. Nonindependent K+ movement through the pore in IRK1 potassium channels. J Gen Physiol 112 475-84 (1998).

17. Heginbotham, L. \& MacKinnon, R. Conduction properties of the cloned Shaker K+ channel. Biophys J 65, 2089-96 (1993).

18. Macri, V. \& Accili, E. A. Structural elements of instantaneous and slow gating in hyperpolarization-activated cyclic nucleotide-gated channels. J Biol Chem $\mathbf{2 7 9}$, 16832-46 (2004)

19. DiFrancesco, D. A new interpretation of the pace-maker current in calf Purkinje fibres. J Physiol 314, 359-76 (1981).

20. Wollmuth, L. P. \& Hille, B. Ionic selectivity of Ih channels of rod photoreceptors in tiger salamanders. J Gen Physiol 100, 749-65 (1992).

21. D’Avanzo, N., Pekhletski, R. \& Backx, P. H. P-loop residues critical for selectivity in K channels fail to confer selectivity to rabbit HCN4 channels. PLoS One 4, e7712 (2009).

22. Doyle, D. A. et al. The structure of the potassium channel: molecular basis of $\mathrm{K}+$ conduction and selectivity. Science 280, 69-77 (1998).

23. DiFrancesco, D. Characterization of single pacemaker channels in cardiac sinoatrial node cells. Nature 324, 470-3 (1986).

24. Dekker, J. P. \& Yellen, G. Cooperative gating between single HCN pacemaker channels. J Gen Physiol 128, 561-7 (2006).

25. Giorgetti, A., Carloni, P., Mistrik, P. \& Torre, V. A homology model of the pore region of HCN channels. Biophys J 89, 932-44 (2005).

26. Rothberg, B. S., Shin, K. S., Phale, P. S. \& Yellen, G. Voltage-controlled gating at the intracellular entrance to a hyperpolarization-activated cation channel. J Gen Physiol 119, 83-91 (2002).

27. Shin, K. S., Rothberg, B. S. \& Yellen, G. Blocker state dependence and trapping in hyperpolarization-activated cation channels: evidence for an intracellular activation gate. J Gen Physiol 117, 91-101 (2001).

28. Roncaglia, P., Mistrik, P. \& Torre, V. Pore topology of the hyperpolarizationactivated cyclic nucleotide-gated channel from sea urchin sperm. Biophys $J$ 83, 1953-64 (2002).

29. Shealy, R. T., Murphy, A. D., Ramarathnam, R., Jakobsson, E. \& Subramaniam, S Sequence-function analysis of the $\mathrm{K}+$-selective family of ion channels using a comprehensive alignment and the KcsA channel structure. Biophys J 84, 2929-42 (2003).

30. Yu, F. H. \& Catterall, W. A. The VGL-chanome: a protein superfamily specialized for electrical signaling and ionic homeostasis. Sci STKE 2004, re15 (2004).

31. Jiang, Y. et al. X-ray structure of a voltage-dependent K+ channel. Nature $\mathbf{4 2 3}$, $33-41$ (2003)

32. Aqvist, J. \& Luzhkov, V. Ion permeation mechanism of the potassium channel. Nature 404, 881-4 (2000).

33. Derebe, M. G. et al. Tuning the ion selectivity of tetrameric cation channels by changing the number of ion binding sites. Proc Natl Acad Sci US A 108, 598-602.

34. Zhou, M. \& MacKinnon, R. A mutant KcsA K(+) channel with altered conduction properties and selectivity filter ion distribution. J Mol Biol 338, 839-46 (2004)
35. Sauer, D. B., Zeng, W., Raghunathan, S. \& Jiang, Y. Protein interactions central to stabilizing the $\mathrm{K}+$ channel selectivity filter in a four-sited configuration for selective K+ permeation. Proc Natl Acad Sci U S A 108, 16634-9.

36. Proenza, C., Angoli, D., Agranovich, E., Macri, V. \& Accili, E. A. Pacemaker channels produce an instantaneous current. J Biol Chem 277, 5101-9 (2002).

37. Cunningham, B. C. \& Wells, J. A. High-resolution epitope mapping of hGHreceptor interactions by alanine-scanning mutagenesis. Science $\mathbf{2 4 4}, 1081-5$ (1989).

38. Andalib, P., Wood, M. J. \& Korn, S. J. Control of outer vestibule dynamics and current magnitude in the Kv2.1 potassium channel. J Gen Physiol 120, 739-55 (2002).

39. Hestrin, S. The properties and function of inward rectification in rod photoreceptors of the tiger salamander. J Physiol 390, 319-33 (1987).

40. Edman, A. \& Grampp, W. Ion permeation through hyperpolarization-activated membrane channels (Q-channels) in the lobster stretch receptor neurone. Pflugers Arch 413, 249-55 (1989).

41. Frace, A. M., Maruoka, F. \& Noma, A. External K+ increases Na + conductance of the hyperpolarization-activated current in rabbit cardiac pacemaker cells. Pflugers Arch 421, 97-9 (1992).

42. Heginbotham, L., Lu, Z., Abramson, T. \& MacKinnon, R. Mutations in the K+ channel signature sequence. Biophys J 66, 1061-7 (1994).

43. Bichet, D., Grabe, M., Jan, Y. N. \& Jan, L. Y. Electrostatic interactions in the channel cavity as an important determinant of potassium channel selectivity. Proc Natl Acad Sci U S A 103, 14355-60 (2006).

44. Bichet, D. et al. Evolving potassium channels by means of yeast selection reveals structural elements important for selectivity. Proc Natl Acad Sci U S A 101, 4441-6 (2004).

45. Grabe, M., Bichet, D., Qian, X., Jan, Y. N. \& Jan, L. Y. K+ channel selectivity depends on kinetic as well as thermodynamic factors. Proc Natl Acad Sci U S A 103, 14361-6 (2006).

46. Alam, A. \& Jiang, Y. Structural analysis of ion selectivity in the NaK channel. Nat Struct Mol Biol 16, 35-41 (2009).

\section{Acknowledgements}

VM was supported by a Doctoral Research Award from the Canadian Institutes of Health Research and the Michael Smith Foundation for Health Research. This study was also supported by a Grant-in-Aid from the Heart and Stroke Foundation of British Columbia and Yukon (EA). EA is also the recipient of a Tier II Canada Research Chair.

\section{Author contributions}

VM, DA and EA conceived the experiments. VM and DA performed the experiments and, together with EA, analyzed and interpreted the results. VM and EA wrote most of the manuscript and prepared the figures, while DA initially wrote small sections and helped to prepare some of the figures. Manuscript was edited by VM and EA.

\section{Additional information}

Supplementary information accompanies this paper at http://www.nature.com/ scientificreports

Competing financial interests The authors declare no competing financial interests.

License: This work is licensed under a Creative Commons

Attribution-NonCommercial-NoDerivs 3.0 Unported License. To view a copy of this license, visit http://creativecommons.org/licenses/by-nc-nd/3.0/

How to cite this article: Macri, V., Angoli, D. \& Accili, E.A. Architecture of the HCN selectivity filter and control of cation permeation. Sci. Rep. 2, 894; DOI:10.1038/srep00894 (2012) 